\title{
Preliminary Cytotoxicity Screening of Some Medicinal Plants of Bangladesh
}

\section{Mohammad S. Rahman ${ }^{1}$, Bilkis Begum ${ }^{2}$, Rasheduzzaman Chowdhury ${ }^{1}$, Khondaker M. Rahman ${ }^{1}$ and Mohammad A. Rashid ${ }^{1,3}$}

\author{
${ }^{1}$ Department of Pharmaceutical Chemistry, Faculty of Pharmacy, University of Dhaka, \\ Dhaka-1000, Bangladesh \\ ${ }^{2}$ Department of Clinical Pharmacy and Pharmacology, Faculty of Pharmacy, University of Dhaka, \\ Dhaka-1000, Bangladesh \\ ${ }^{3}$ Centre for Biomedical Research, University of Dhaka, Dhaka-1000, Bangladesh
}

\begin{abstract}
The cytotoxic activity of the methanolic extracts of 35 plant species, including 28 traditionally used plants of Bangladesh was evaluated by the brine shrimp lethality bioassay technique. Among these, 19 plant extracts exhibited significant toxicity to brine shrimps with $\mathrm{LC}_{50}$ less than $10 \mu \mathrm{g} / \mathrm{ml}$.
\end{abstract}

Key words: Cytotoxic activity, Brine shrimp lethality bioassay.

\section{INTRODUCTION}

Plants are the natural reservoir of many antimicrobial and anticancer agents. Bangladeshi people have traditional medical practice as an integral part of their culture. A lot of medicinal plants are available for the treatment of various diseases. However, scientific studies have been conducted only to a limited extent with few medicinal plants. ${ }^{1-4}$ In this investigation, 35 locally used plants were selected and tested to justify their existing bioactivities by the brine shrimp lethality bioassay. ${ }^{5}$ The method utilizes in vivo lethality in a simple zoological organism brine shrimp nauplii as a convenient monitor for screening cytotoxicity of the plant extracts which can be furhter correlated with its anticancer potentiality and other bioactivities.

Correspondence to: Mohammad A. Rashid Tel.: 880-2-8612069, 9661900-73, extn.- 4363, 4364, 8137 Fax: 880-2-8612069

E-mail: rashidma@aitlbd.net

\section{MATERIALS AND METHODS}

Plant collection. The plants selected for the study (Table 1) were collected from Dhaka, Chittagong and Khulna districts of Bangladesh during January - April 2004 and identified at the Department of Botany, University of Dhaka where voucher specimens for these collections are maintained.

Extraction. The air-dried and powdered plant materials were separately extracted with methanol for 5 days at room temperature with occasional shaking and stirring. The extracts were then filtered off through a cotton plug and finally with Whatman no.1 filter papers. The volume of the filtrate was reduced using a Buchii rotary evaporator at low temperature and pressure.

Bioassay. Brine shrimp lethality bioassay ${ }^{5}$ technique was applied for the determination of cytotoxic property of the plant extractives. Vincristine sulphate and DMSO were used as positive and negative control, respectively. 
Table 1. LC $_{50}$ of methanol extracts of some medicinal plants of Bangladesh

\begin{tabular}{|c|c|c|c|c|}
\hline Plant (Family) & $\begin{array}{l}\text { Uses in traditional } \\
\text { medicine }\end{array}$ & RMC & RPPS & $\begin{array}{c}\mathrm{LC}_{50} \\
(\mu \mathrm{g} / \mathrm{ml})\end{array}$ \\
\hline $\begin{array}{l}\text { Aglaia roxburghiana } \\
\text { (Meliaceae) }\end{array}$ & $\begin{array}{l}\text { Dysentry, leucoderma, } \\
\text { leprosy, fever, thirst, } \\
\text { tumors, vomiting. }\end{array}$ & $\begin{array}{l}\text { 24, 25-epoxy-29-norcycloartan-3-ol, 29- } \\
\text { norcyclorart-23-ene-3, 25-diol , 24,25-epoxy-29- } \\
\text { nor-24-cycloarten-3 } \beta \text {-ol, roxburghiline, } \\
\text { hydroxyroxburghiline, aglaroxin-A, } \\
\text { roxhurghiadiol A. }\end{array}$ & $\begin{array}{l}\text { Terpenoids, } \\
\text { alkaloids }\end{array}$ & $11.66 \pm 1.10$ \\
\hline $\begin{array}{l}\text { Amoora cucullata } \\
\text { (Meliaceae) }\end{array}$ & Inflammation. $^{6}$ & No information found. & Steroids & $5.16 \pm 1.13$ \\
\hline $\begin{array}{l}\text { Amoora rohituka } \\
\text { (Meliaceae) }\end{array}$ & $\begin{array}{l}\text { Cancer, tumours, spleen } \\
\text { and liver disease, } \\
\text { rheumatism. }^{7}\end{array}$ & $\begin{array}{l}\text { 6b,7b-epoxyguai-4-en-3-one, } 6 \mathrm{~b}, 7 \mathrm{~b} \text {-epoxy- } 4 \mathrm{~b}, 5 \text { - } \\
\text { dihydroxyguaiane, }{ }^{11} \text { stigmasta-5,24(28)-dien-3 } 3 \text { - } \\
\text { O- } \beta \text {-D-glucopyranosyl-O- } \alpha \text {-L- } \\
\text { rhamnopyranoside, }{ }^{12} \text { 7-keto-octadec-cis-I 1-enoic } \\
\text { acid. }^{13}\end{array}$ & Terpenoids & $5.95 \pm 0.94$ \\
\hline $\begin{array}{l}\text { Brunfelsia americana } \\
\text { (Solanaceae) }\end{array}$ & No information found. & No information found. & Steroids & $7.43 \pm 1.17$ \\
\hline $\begin{array}{l}\text { Brunfelsia latifolia } \\
\text { (Solanaceae) }\end{array}$ & No information found. & No information found. & Steroids & $6.82 \pm 0.47$ \\
\hline $\begin{array}{l}\text { Buchanania lanzan } \\
\text { (Anacardiaceae) }\end{array}$ & $\begin{array}{l}\text { fever, thirst, diarrhea, } \\
\text { itch. }^{8}\end{array}$ & Myricetin 3'-rhamnoside-3-galactoside. ${ }^{14}$ & flavonoid & $63.42 \pm 1.34$ \\
\hline $\begin{array}{l}\text { Chukrasia tabularis } \\
\text { (Meliaceae) }\end{array}$ & $\begin{array}{l}\text { As an astringent and } \\
\text { antidiarrhoeic. }^{6}\end{array}$ & $\begin{array}{l}\text { tabulalides A-E },{ }^{15} \text { tabularin, }{ }^{16} \text { scopoletin, } \\
\text { melianone, }{ }^{17} \text { chukrasin A-E. }{ }^{10}\end{array}$ & $\begin{array}{l}\text { Terpenoids, } \\
\text { coumarins }\end{array}$ & $15.16 \pm 1.27$ \\
\hline $\begin{array}{l}\text { Combretum coccineum } \\
\text { (Combretaceae) }\end{array}$ & No information found. & A hydroxylated mansumbinen-28-oic acid. ${ }^{18}$ & Terpenoids & $5.95 \pm 0.37$ \\
\hline $\begin{array}{l}\text { Erioglossum edule } \\
\text { (Sapindaceae) }\end{array}$ & $\begin{array}{l}\text { Fever, whooping } \\
\text { cough. }^{6}\end{array}$ & No information found. & Steroids & $158.74 \pm 1.24$ \\
\hline $\begin{array}{l}\text { Ficus indica } \\
\text { (Moraceae) }\end{array}$ & $\begin{array}{l}\text { Relieve toothache, } \\
\text { rheumatism, lumbago, } \\
\text { inflammations, } \\
\text { diarrhoea, dysentry, } \\
\text { vomitting, biliousness. }\end{array}$ & $\begin{array}{l}\text { Bengalenoside, leucoanthocyanidins, } \\
\text { leucoanthocyanin glycoside, betasitusterol } \\
\text { glycoside, mesoinositol, friedelin, beta-sitosterol, } \\
\text { qurecetin-3-galactoside and rutin, tiglic acid ester } \\
\text { of gamma-tarxerol, cyanidin rhamnoglycoside, } \\
\text { ficusin and bergaptin.? }\end{array}$ & $\begin{array}{l}\text { Steroids, } \\
\text { terpenoids, } \\
\text { phenolics }\end{array}$ & $17.67 \pm 1.17$ \\
\hline $\begin{array}{l}\text { Garuga pinnata } \\
\text { (Burseraceae) }\end{array}$ & $\begin{array}{l}\text { Asthma, opacity of } \\
\text { conjucntiva. }\end{array}$ & $\begin{array}{l}\text { Garuganins I-VI, eupha-7, 24-diene-1, 3- diol, } \\
\text { eupha-7, 24-diene-3, 11, 16-triol. }{ }^{10}\end{array}$ & Terpenoids & $37.72 \pm 3.10$ \\
\hline $\begin{array}{l}\text { Indigofera tinctoria } \\
\text { (Papilionaceae) }\end{array}$ & $\begin{array}{l}\text { Epilepsy, nervous } \\
\text { disorder, bronchitis, } \\
\text { sores, old ulcers. }\end{array}$ & Galactomannan [19], indirubin. $^{20}$ & $\begin{array}{l}\text { Steroids, } \\
\text { terpenoids }\end{array}$ & $1.86 \pm 1.14$ \\
\hline $\begin{array}{l}\text { Lannea coromandelica } \\
\text { (Anacardiaceae) }\end{array}$ & $\begin{array}{l}\text { Leprous and obstinate } \\
\text { ulcers, toothache, mouth } \\
\text { sores, impotency. }\end{array}$ & $\begin{array}{l}(2 R, 3 S)-(+)-3^{\prime}, 5 \text {-dihydroxy-4',7- } \\
\text { dimethoxydihydroflavonol, }(2 R, 3 R)-(+)-4^{\prime}, 5,7- \\
\text { trimethoxydihydroflavonol, }(2 \mathrm{R}, 3 \mathrm{R})-(+)-4^{\prime}, 7-\mathrm{di}- \\
\text { O-methyldihydroquercetin, }(2 \mathrm{R}, 3 \mathrm{R})-(+)-4^{\prime}, 7-\mathrm{di}- \\
\text { O-methyldihydrokaempferol and }(2 \mathrm{R}, 3 \mathrm{R})-(+)-4^{\prime}- \\
\text { O-methyldihydroquercetin }[21], \text { Quercetin-3- } \\
\text { arabinoside, ellagic acid, } \beta \text {-sitosterol, physcion, } \\
\text { physcion anthranol B, leucocyanidin. }\end{array}$ & Phenolics & $53.59 \pm 1.33$ \\
\hline $\begin{array}{l}\text { Nephelium litchi } \\
\text { (Sapindaceae) } \\
\text { Syn: Litchi chinensis }\end{array}$ & $\begin{array}{l}\text { Neurological disorders, } \\
\text { smallpox, throat } \\
\text { infection. }{ }^{7}\end{array}$ & $\begin{array}{l}\text { Folic acid, L - ascorbic acid, cyanidin-3- } \\
\text { glucoside, cyanidin-3-rutinoside, malvidin-3- } \\
\text { acetylglucoside, } \\
\alpha \text {-[methylene cyclopropyl] glycine. }\end{array}$ & $\begin{array}{l}\text { Steroids, } \\
\text { terpenoids }\end{array}$ & $143.98 \pm 1.64$ \\
\hline $\begin{array}{l}\text { Nephelium longana } \\
\text { (Sapindaceae) } \\
\text { Syn: Euphoria longan }\end{array}$ & Anthelmintic, fever. ${ }^{6}$ & $\begin{array}{l}\text { Saponin, }{ }^{6} \text { 2-amino-4-methylhex-5-ynoic acid, } 2 \text { - } \\
\text { amino-4-hydroxymethylhex-5-ynoic acid, 2- } \\
\text { amino-4-hydroxyhept-6-ynoic acid. }\end{array}$ & $\begin{array}{l}\text { Steroids, } \\
\text { terpenoids, } \\
\text { amino acids. }\end{array}$ & $2.21 \pm 0.66$ \\
\hline $\begin{array}{l}\text { Passiflora coccinea } \\
\text { (Passifloraceae) }\end{array}$ & No information found. & Passicoccin. $^{24}$ & $\begin{array}{l}\text { Cyanogenic } \\
\text { glycoside }\end{array}$ & $131.95 \pm 1.33$ \\
\hline
\end{tabular}


Table 1 (Contd.)

\begin{tabular}{|c|c|c|c|c|}
\hline Plant (Family) & $\begin{array}{l}\text { Uses in traditional } \\
\text { medicine }\end{array}$ & RMC & RPPS & $\begin{array}{c}\mathrm{LC}_{50} \\
(\mu \mathrm{g} / \mathrm{ml})\end{array}$ \\
\hline $\begin{array}{l}\text { Petunia meleagris } \\
\text { (Solanaceae) }\end{array}$ & No information found. & No information found. & Steroids & $53.59 \pm 2.31$ \\
\hline $\begin{array}{l}\text { Petunia phoenica } \\
\text { (Solanaceae) }\end{array}$ & No information found. & No information found. & Steroids & $30.31 \pm 1.28$ \\
\hline $\begin{array}{l}\text { Petunia punctata } \\
\text { (Solanaceae) }\end{array}$ & No information found. & No information found. & Steroids & $4.90 \pm 1.64$ \\
\hline Petunia violaceae & No information found. & No information found. & Steroids & $41.14 \pm 1.67$ \\
\hline $\begin{array}{l}\text { Phyllanthus reticulatus } \\
\text { (Euphorbiaceae) }\end{array}$ & $\begin{array}{l}\text { Spongy and bleeding } \\
\text { gums, as astringent, } \\
\text { diuretic. }^{6}\end{array}$ & $\begin{array}{l}\text { Friedelin, sitosterol, friedelan-38-02 glochidonol, } \\
\text { 2la-hydroxyfriedelan3-one, } 21 \text { a-hydroxyfriedel- } \\
\text { 4(23)-en-3-one, betulinic acid. }{ }^{25}\end{array}$ & $\begin{array}{l}\text { Terpenoids, } \\
\text { steroids }\end{array}$ & $3.72 \pm 1.98$ \\
\hline \multirow[t]{2}{*}{$\begin{array}{l}\text { Pongamia glabra } \\
\text { (Leguminosae) }\end{array}$} & \multirow[t]{2}{*}{$\begin{array}{l}\text { Bleeding piles, fistulous } \\
\text { sores, bronchitis, } \\
\text { gonorrhoea, whooping } \\
\text { cough, } \\
\text { as tonic. }\end{array}$} & $\begin{array}{l}\text { Karanjin, ovalitenone, pongachromene, } \\
\text { lanceolatin, betulinic acid, caffeic esters, } \\
\text { pongapin, glabrachromene, desmethoxykanugin, } \\
\text { (-)-isoglabrachromene, kanugin, glabra-ii, fisetin } \\
\text { tetramethyl ether, 5-methoxy-3',4'- } \\
\text { methylenedioxy-2", 2"'-do(7,8-6",5") flavone, }{ }^{26} \\
\text { glabone. }{ }^{27} \text { pongagallone-a, pongagallone-b, }{ }^{28} \\
\text { isopongachromene, pongamol, kanjone, } \\
\text { pongaglabrone, }{ }^{29}\end{array}$ & \multirow[t]{2}{*}{ Flavonoids } & \multirow[t]{2}{*}{$8.54 \pm 1.31$} \\
\hline & & $\begin{array}{l}\text { glabrachalcone, }{ }^{30} \text { isopongaglabol and 6- } \\
\text { methoxyisopongaglabol, 5- } \\
\text { methoxyfurano( } 8,74 ", 5 ") \text { flavone, } 5 \text {-methoxy- } \\
3^{\prime}, 4^{\prime} \text {-methylenedioxyfurano( }(8,7-4 ", 5 \text { ")flavone, } \\
\text { ovalichromene B, cycloart-23-ene-3p,25-diol, } \\
\text { friedelin, and } \beta \text {-sitosterol- } \beta \text {-D-glucoside, }{ }^{31} \\
\text { pongaglabol, aurantiamide acetate }{ }^{32} \\
\text { pongaglabrone. }\end{array}$ & & \\
\hline \multirow{5}{*}{$\begin{array}{l}\text { Pterospermum } \\
\text { suberifolium } \\
\text { (Sterculiaceae) }\end{array}$} & \multirow[t]{5}{*}{ Smallpox, hemicrania. $^{6}$} & 3,4-Di-O-methylrhamnose & \multirow[t]{5}{*}{ Phenolics } & \multirow[t]{5}{*}{$2.48 \pm 0.88$} \\
\hline & & 2,3,4,6-Tetra-O-methylglucose & & \\
\hline & & 2,3,6-Tri-O-methylglucose & & \\
\hline & & 2,3,4,6-Tetra-O-methylgalactose & & \\
\hline & & $\begin{array}{l}\text { 2,3,6-Tri-O-methylgalactose, } 4,6 \text {-Di-O- } \\
\text { methylgalactose } 3,6 \text {-Di-O-methylgalactose. }\end{array}$ & & \\
\hline $\begin{array}{l}\text { Quisqualis indica } \\
\text { (Combretaceae) }\end{array}$ & $\begin{array}{l}\text { Diarrhea, fever, rickets } \\
\text { in children, boils, } \\
\text { ulcers, helminthiasis. }\end{array}$ & Quisqualic acid, ${ }^{35}$ quisqualin A. ${ }^{10}$ & $\begin{array}{l}\text { Amino acids, } \\
\text { tannins }\end{array}$ & $4.42 \pm 1.57$ \\
\hline \multirow[t]{2}{*}{$\begin{array}{l}\text { Semecarpus } \\
\text { anacardium } \\
\text { (Anacardiaceae) }\end{array}$} & \multirow[t]{2}{*}{$\begin{array}{l}\text { Scrofulous, venereal } \\
\text { and leprous infections, } \\
\text { nervous debility. }\end{array}$} & $\begin{array}{l}\text { Anacardic acid, semicarpol, bhilawanol, } \\
\text { monolefin I, diolefin II, bhilawanol-A, } \\
\text { bhilawanol-B, amentoflavone } \\
\text { tetrahydroamentoflavone, tetrahydrobustaflavone, } \\
\text { jeediflavanone, } \\
\text { semecarpuflavanone,galluflavanone } \\
\text { anacarduflavone, anacardoside, }{ }^{36} \text { semecarpetin, } \\
\text { nallaflavanone, }{ }^{37} \text { jeediflavanone, }{ }^{38} \\
\text { semecarpuflavanone, }{ }^{39} \text { galluflavanone }{ }^{40} \mathrm{O}- \\
\text { trimethyl biflavanone } \mathrm{A}_{1}, \mathrm{O} \text {-trimethyl biflavanone } \\
\mathrm{A}_{2} \text {, O-Tetramethyl biflavanone } \mathrm{A}_{1}, \mathrm{O} \text {-hexamethyl } \\
\text { bichalcone A, }\end{array}$ & & \\
\hline & & $\begin{array}{l}\text { O-dimethyl biflavonone } \mathrm{B} \text {, O-heptamethyl } \\
\text { bichalcone } \mathrm{B}_{1} \text {, O-hexamethyl bichalcone } \mathrm{B}_{2} \text {, O- } \\
\text { tetramethyl biflavanone } \mathrm{C}^{41}\end{array}$ & Phenolics & $35.36 \pm 0.94$ \\
\hline $\begin{array}{l}\text { Shorea robusta } \\
\text { (Dipterocarpaceae) }\end{array}$ & $\begin{array}{l}\text { Ulcers, wounds, } \\
\text { gonorrhea, leprosy, } \\
\text { helminthiasis. }^{8}\end{array}$ & $\begin{array}{l}\text { 9,10-dihydroxystearic acid, 3,25-epoxy-1,2,3,11- } \\
\text { tetrahydroxy-12-ursen-28-oic acid, } 28 \text {-nor-12- } \\
\text { ursen-3-ol, shorea phenol, } 2,3,23 \text {-trihydroxy-11- } \\
\text { methoxy-12-ursen-28-oic acid. }{ }^{10}\end{array}$ & Terpenoids & $3.50 \pm 1.38$ \\
\hline
\end{tabular}


Table 1 (Contd.)

\begin{tabular}{|c|c|c|c|c|}
\hline Plant (Family) & $\begin{array}{l}\text { Uses in traditional } \\
\text { medicine }\end{array}$ & $\mathrm{RMC}$ & RPPS & $\begin{array}{c}\mathrm{LC}_{50} \\
(\mu \mathrm{g} / \mathrm{ml})\end{array}$ \\
\hline $\begin{array}{l}\text { Solamum indicum } \\
\text { (Solanaceae) }\end{array}$ & $\begin{array}{l}\text { Astringent, carminative, } \\
\text { cardiac tonic, } \\
\text { aphrodisiac. }^{6}\end{array}$ & $\begin{array}{l}\text { Isoanguivine, protodioscin, solasonine and } \\
\text { solamargine, indioside A-E. }\end{array}$ & Steroids & $4.42 \pm 0.67$ \\
\hline $\begin{array}{l}\text { Solanum ferox } \\
\text { ( Solanaceae) }\end{array}$ & $\begin{array}{l}\text { Coughs, asthma, fever, } \\
\text { vomiting, sore throat, } \\
\text { gonorrhea. }{ }^{6}\end{array}$ & Carpesterol, solanocarpine. $^{6}$ & $\begin{array}{l}\text { Steroids, } \\
\text { alkaloids }\end{array}$ & $12.92 \pm 1.49$ \\
\hline $\begin{array}{l}\text { Spondias mangifera } \\
\text { (Anacardiaceae) }\end{array}$ & $\begin{array}{l}\text { Antiscorbutic, } \\
\text { astringent, dysentry, } \\
\text { diarrhoea, vomitting, } \\
\text { gonorrhoea and } \\
\text { leuchoohoea. }\end{array}$ & $\begin{array}{l}\text { 3, 16-dihydroxy-12-oleanen-28-oic acid -3-O- }[\beta- \\
\text { D-galactopyranosyl-(1-->5) xylopyranoside }] .{ }^{10}\end{array}$ & Terpenoids & $4.42 \pm 0.99$ \\
\hline $\begin{array}{l}\text { Swintonnia floribunda } \\
\text { (Anacardiaceae) }\end{array}$ & No information found. & No information found. & Steroids & $32.66 \pm 1.68$ \\
\hline \multirow[t]{2}{*}{$\begin{array}{l}\text { Terminalia bellirica } \\
\text { (Combretaceae) }\end{array}$} & $\begin{array}{l}\text { Hepatitis, breathing } \\
\text { problem, coughs, eye } \\
\text { disease, constipation } \\
\text { purgative. }\end{array}$ & $\begin{array}{l}\text { Cardenolide }{ }^{43} 2 \text {-dotriacontanol, bellericagenin B, } \\
\text { bellericaside B, termilignan, bellericagenin A, } \\
\text { bellericaside A, thannilignan, 9-tritriacontanone, } \\
\text { sitosterol, gallic aad, ellagic, acid, ethyl gallate, } \\
\text { galloyl glucose, mannitol, glucose, galactose, } \\
\text { fructose }\end{array}$ & & \\
\hline & & $\begin{array}{l}\text { and rhamnose, bellerlc acid, bellericoside, } \\
\text { arjungenin, arjunglycoside. }{ }^{44}\end{array}$ & $\begin{array}{l}\text { Terpenoids, } \\
\text { phenolics, } \\
\text { steroids, }\end{array}$ & $3.62 \pm 1.35$ \\
\hline $\begin{array}{l}\text { Terminalia chebula } \\
\text { (Combretacacae) }\end{array}$ & $\begin{array}{l}\text { Indigestion, } \\
\text { constipation } \\
\text { jaundice, piles, painful } \\
\text { menstruation, asthma, } \\
\text { colic, as diuretic and } \\
\text { cardiotonic. }\end{array}$ & $\begin{array}{l}\text { Terflavin A, chebulagic acid, chebulinic acid, } \\
\text { corilagin, } 2 \alpha \text {-hydroxymicromeric acid, luteolic } \\
\text { acid, 12-oleanene- } 2,3,19,23,28 \text {-pentol, terchebin, } \\
\text { terchebulin, terflavin } \mathrm{D} \text {, terfalvin } \mathrm{B}, 1,3,6- \\
\text { trigalloyl glucose. }\end{array}$ & Phenolics & $8.85 \pm 0.38$ \\
\hline $\begin{array}{l}\text { Trachyspermum ammi } \\
\text { (Umbelliferae) }\end{array}$ & $\begin{array}{l}\text { Diarrhea, colic, } \\
\text { flatulence, indigestion, } \\
\text { cholera, dyspepsia. }\end{array}$ & An essential oil containing thymol. ${ }^{6}$ & Terpenoids & $4.95 \pm 1.61$ \\
\hline $\begin{array}{l}\text { Xylocarpus granatum } \\
\text { (Meliaceae) }\end{array}$ & $\begin{array}{l}\text { Dysentery, diarrhea, and } \\
\text { other abdominal } \\
\text { problems. }\end{array}$ & $\begin{array}{l}\text { xyloccensins O-P, xyloccensins Q-V, } \\
\text { Xyloccensin L, }{ }^{46} \text { xyloccensin K, }{ }^{47} \text { xyloccensin I- } \\
\mathrm{J}^{48}\end{array}$ & Terpeonoids & $6.81 \pm 0.22$ \\
\hline $\begin{array}{l}\text { Zizyphus mauritiana } \\
\text { (Rhamnaceae) }\end{array}$ & $\begin{array}{l}\text { Diarrhea, fever, } \\
\text { delirium, gout, wounds } \\
\text { and ulcers. }\end{array}$ & $\begin{array}{l}\text { Mauritine A-H, frangufoline, amphibine B, } \\
\text { amphibine D-F, }{ }^{49} \text { zizogenin, }{ }^{50} \text { laccaic acid D. }{ }^{10}\end{array}$ & Alkaloids & $22.75 \pm 0.34$ \\
\hline
\end{tabular}

$\mathrm{RMC}$, repoted major constituents; RPPS, results of preliminary phytochemical screenings; $\mathrm{LC}_{50}, 50 \%$ of lethal concentration; $\mathrm{LC}_{50}$ were calculated as mean $\pm \mathrm{SD}(\mathrm{n}=3)$.

$4 \mathrm{mg}$ of each of the extractives was dissolved in DMSO and solutions of varying concentrations such as $400,200,100,50,25,12.50,6.25,3.125,1.563$ and $0.781 \mu \mathrm{g} / \mathrm{ml}$ were obtained by serial dilution technique. Then the solutions were added to the premarked vials containing ten live brine shrimp nauplii in $5 \mathrm{ml}$ simulated sea water. After 24 hours, the vials were inspected using a magnifying glass and the number of survived nauplii in each vial was counted. From this data, the percent (\%) of lethality of the brine shrimp nauplii was calculated for each concentration. The median lethal concentration
$\left(\mathrm{LC}_{50}\right)$ of the test samples was obtained by a plot of percentage of the shrimps killed against the logarithm of the sample concentration.

Statistical analysis. The experiment was conducted in triplicate and the $\mathrm{LC}_{50}$ were calculated as mean $\pm \mathrm{SD}(\mathrm{n}=3)$.

\section{RESULTS AND DISCUSSION}

In the present study, extracts of 35 local plants used in Bangladesh were evaluated by the brine shrimp lethality bioassay using the procedure 
designed by Meyer et al. ${ }^{5}$ The $\mathrm{LC}_{50}$ values of the brine shrimp assay obtained for extracts of these medicinal plants are listed in Table 1.

The methanolic extract of Indigofera tinctoria showed the highest activity with $\mathrm{LC}_{50} 1.86 \mu \mathrm{g} / \mathrm{ml}$. The extracts of Nephelium longan, Pterospermum suberifolium, Shorea robusta, Terminalia bellerica and Phyllanthus reticulatus exhibited strong brine shrimp lethality with $\mathrm{LC}_{50}$ values of $2.21,2.48,3.50$, 3.62 and $3.72 \mu \mathrm{g} / \mathrm{ml}$, respectively. In addition, Solanum indicum, Quisqualis indica, Spondias mangifera, Petunia punctata, Trachyspermum ammi, Amoora cucullata, Amoora rohituka and Combretum coccineum have also shown significant brine shrimp lethality and the $\mathrm{LC}_{50}$ values were found to be lower than 6.00 (Table 1). On the otherhand, Xylocarpus grantum, Brunfelsia latifolia, Brunfelsia americana, Pongamia glabra and Terminalia chebula showed moderate cytotoxicity with $\mathrm{LC}_{50}$ less than 10.00 $\mu \mathrm{g} / \mathrm{ml}$.

It is observed that 19 plants extracts were highly lethal to brine shrimp nauplii out of the 35 plants used in the study. This indicates that these plants contain potential bioactive compounds, which if properly and extensively studied, could provide many chemically interesting and biologically active drug candidates, including some with potential antitumor and antiproliferative properties. A thorough chemical study is required to isolate the molecules that are responsible for the activities.

\section{ACKNOWLEDGEMENT}

We wish to thank the Ministry of Science and information \& Communication Technology, Government of the Peoples' Republic of Bangladesh for partial financial support to carry out the research.

\section{REFERENCES}

1. Rashid, M.A., Hasan, C.M., Choudhury, S.A.R., Begum, B. and Rahman, S. 1997. Ethnopharmacological investigation of medicinal plants of Bangladesh. Bangladesh Journal of Physiology and Pharmacology 12, 25-29.
2. Nutan, M.T.H., Hasnat, A., Rashid, M.A. and Rahman, S. 1997. Cytotoxic and antiproliferative medicinal plants of Bangladesh a review Bangladesh. Journal of Life Sciences 9, 61-67.

3. Haque, N., Choudhury, S.A.R., Nutan, M.T.H., Rahman, G.D.S. and Rashid, M.A. 2000. Antibacterial screening of some medicinal plants of Bangladesh. Bangladesh Journal of Physiology and Pharmacology 15, 52-54.

4. Haque, N., Choudhury, S.A.R., Nutan, M.T.H., Rahman, G.D.S. and Rashid, M.A. 2000. Evaluation of antitumor activity of some medicinal plants of Bangladesh by potato disk bioaasay. Fitoterapia 71, 544-549.

5. Meyer, B.N., Ferringni, N.R., Puam, J.E., Lacobsen, L.B., Nichols, D.E. and McLaughlin JL. 1982. Brine shrimp: a convenient general bioassay for active constituents. Planta Medica 45, 31-32.

6. Chopra, R.N., Nayar, S.L. and Chopra, I.C. 1956. Glossary of Indian Medicinal Plants, New Delhi, CSIR.

7. Ghani, A.1998. Medicinal plants of Bangladesh. 2nd ed. Dhaka; Asiatic Society of Bangladesh.

8. Kirtikar, K.R., Basu, B.D., Blatter, E., Caius, J.F. and Mahaskar, K.S. 1980. Indian medicinal plants. 2nd ed. Singh B \& Singh MP publishers.

9. Yusuf, M., Chowdhury, J.U., Wahab, M.A. and Begum, J. 1994. Medicinal Plants of Bangldesh. 1st ed. Dhaka; Bangladesh Council of Scientific and Industrial Research (BCSIR).

10. Dictionary of Natural Products, Chapman and Hall, 2002.

11. Chowdhury, R., Hasan, C.M. and Rashid, M.A. 2003. Guaiane sesquiterpenes from Amoora rohituka. Phytochemistry 62, 1213-1216.

12. Bhatt, S.K., Saxena, V.K. and Nigam, S.S. 1981. A new saponin from seeds of Amoora rohituka. Phytochemistry 20, 1749-1750.

13. Daulatabad, C.D. and Jamkhandi, S.A.M. 1997. A keto fatty acid from Amoora rohituka seed oil. Phytochemistry 46, 155156.

14. Arya, R., Babu, V., Ilyas, M. and Nasim, K.T. 1992. Myricetin 3'-rhamnoside-3-galactoside from Buchanania lanzan (anacardiaceae). Phytochemistry 31, 2569-2570.

15. Nakatani, M., Abdelgaleil, S.A.M., Mona, M.G., Saad, M.M.G., Huang, R.C., Doe, M. and Iwagawa, T. 2004. Phragmalin limonoids from Chukrasia tabularis. Phytochemistry 65, 2833-2841.

16. Purushothaman, K.K., Sarada, A., Saraswathi, G. and Connolly, J.D. 1977. 5,7-dihydroxy-6,2',4',5'-tetramethoxyflavone from the leaves of Chukrasia Tabularis. Phytochemistry 16, 398-399.

17. Chatterjee, A., Banerjee, B., Ganguly, S.N. and Sircar, S.M. 1974. Triterpene and coumarins from Chukrasia tabularis. Phytochemistry 13, 2012-2013. 
18. Chowdhury, R. and Islam, N. A. 2004. Hydroxylated mansumbinen-28-oic acid from Combretum coccineum. Biochemical Systematics and Ecology 32, 443-445.

19. Sen, A.K., Banerjee, N. and Farooqi, M.I.H. 1986. Watersoluble galactomannan from the seeds of Indigofera tinctoria linn. Carbohydrate Research 157, 251-256.

20. Kunikata, T., Tatefuji, T., Aga, H., Iwaki, K., Ikeda, M. and Kurimoto M. 2000. Indirubin inhibits inflammatory reactions in delayed-type hypersensitivity. European Journal of Pharmacology 410, 93-100.

21. Islam, M.T. and Tahara, S. 2000. Dihydroflavonols from Lannea coromandelica. Phytochemistry 54, 901-907.

22. Subramanian, S.S. and Nair, A.G.R. 1971. Polyphenols of Lannea coromandelica. Phytochemistry 10, 1939-1940.

23. Millington, D.S. and Sheppard, R.C. 1969. Acetylenic amino acids from Euphoria longan. Phytochemistry 8, 1227-1233.

24. Spencer, K. and Seigler, D.S. 1985. Passicoccin: a sulphated cyanogenic glycoside from Passiflora coccinea. Phytochemistry 24, 2615-2617.

25. Hui, W., Li, M. and Wong, K. 1976. A new compound, 21 $\alpha-$ hydroxyfriedel-4(23)-en-3-one and other triterpenoids from Phyllanthus reticulatus. Phytochemistry 15, 797-798.

26. Murari, M.S., Uttam, K.M. and Asok K.M. 1991. A chromenoflavanone and two caffeic esters from Pongamia glabra. Phytochemistry 30, 3834-3836.

27. Kanungo, P.D., Ganguly, A., Guha, A., Bhattacharyya, A. and Adityachaudhury N. 1987. Glabone, a new furanoflavone from Pongamia glabra. Phytochemistry 26, 3373-3374.

28. Gandhidasan, R., Neelakantan, S., Raman, P.V. and Devaraj, S. 1986. Components of the galls on the leaves of Pongamia glabra: Structures of pongagallone-a and pongagallone-b. Phytochemistry 26, 281-283.

29. Pathak, V.P., Saini, T.R. and Khanna, R.N. 1983. Isopongachromene, a chromenoflavone from Pongamia glabra seeds. Phytochemistry 22, 308-309.

30. Pathak, V.P., Saini, T.R. and Khanna, R.N. 1983. Glabrachalcone, a chromenochalcone from Pongamia glabra seeds. Phytochemistry 22, 1303-1304.

31. Talapatra, S.K., Mallik, A.K. and Talapatra, B. 1982. Isopongaglabol and 6-methoxyisopongaglabol, two new hydroxyfuranoflavones from Pongamia glabra. Phytochemistry 21, 761-766.

32. Talapatra, S.K., Mallik, A.K. and Talapatra, B. 1980. Pongaglabol, a new hydroxyfuranoflavone, and aurantiamide acetate, a dipeptide from the flowers of Pongamia glabra. Phytochemistry 19, 1199-1202.

33. Khanna, R.N. and Seshadri, T.R. Pongaglabrone, a new component of the seeds of Pongamia glabra: its constitution and synthesis. Tetrahedron 19, 219-225.
34. Pal, N., Ghosh, T.K. and Rao, C.V.N. 1984. Structural and immunochemical studies on Pterospermum Suberifolium gum. Carbohydrate Research 132, 307-315.

35. Shinozaki, H. and Shibuya, I. 1974. A new potent excitant, quisqualic acid: Effects on crayfish neuromuscular junction. Neuropharmacology 13, 665-672.

36. Gil, R.R., Lin, L., Cordell, G.A., Kumar, M.R., Ramesh, M., Reddy, B.M., Mohan, G.K. and Rao, A.V.N.A. 1995. Anacardoside from the seeds of Semecarpus anacardium. Phytochemistry 39, 405-407.

37. Murthy, S.S.N.1988. Semecarpetin, a biflavanone from Semecarpus anacardium. Phytochemistry 27, 3020-3022.

38. Murthy, S.S.N. 1985. Jeediflavanone - a biflavonoid from Semecarpus anacardium. Phytochemistry 24, 1065-1069.

39. Murthy, S.S.N. 1983. A biflavonoid from Semecarpus anacardium. Phytochemistry 22, 1518-1520.

40. Murthy, S.S.N. 1983. A biflavanone from Semecarpus anacardium. Phytochemistry 22, 2636-2638.

41. Rao, N.S.P., Row, L.R. and Brown, R.T. 1973. Phenolic constituents of Semecarpus anacardium. Phytochemistry 12, 671-681.

42. Yahara, S., Nakamura, T., Someya, Y., Matsumoto, T., Yamashita, T. and Nohara, T. 1996. Steroidal glycosides, indiosides A-E, from Solanum indicum. Phytochemistry 43, 1319-1323.

43. Yadava, R.N. and Rathore, K. 2001. A new cardenolide from the seeds of Terminalia bellerica. Fitoterapia 72, 310312 .

44. Nandy, A.K., Podder, G., Niranjan, P., Sahu, N.P. and Mahato SB. 1989. Triterpenoids and their glucosides from Terminalia bellerica. Phytochemistry 28, 2769-2772.

45. Wu, J., Xiao, Q., Zhang, S., Li, X., Xiao, Z., Ding, H. and Li, Q. 2005. Xyloccensins Q-V, six new 8,9,30-phragmalin ortho ester antifeedants from the Chinese mangrove Xylocarpus granatum. Tetrahedron 6, 8382-8389.

46. Wu, J., Zhang, S., Xiao, Q., Li, Q., Huang, J., Long, L. and Huang, L. 2004. Xyloccensin L, a novel limonoid from Xylocarpus granatum. Tetrahedron Letters 45, 591-593.

47. Kokpol, U., Chavasiri, W., Tip-pyang, S., Veerachato, G., Zhao, F., Simpson, J. and Weavers, R.T. 1996. A limonoid from Xylocarpus granatum. Phytochemistry 41, 903-905.

48. Alvi, K.A., Crews, P., Aalbersberg, B. and Prasad, R. 1991. Limonoids from the fijian medicinal plant dabi (xylocarpus). Tetrahedron 4, 8943-8948.

49. Jossang, A., Zahir, A. And Diakite, D. 1996. Mauritine J, a cyclopeptide alkaloid from Zizyphus mauritiana. Phytochemistry 42, 565-567.

50. Srivastava, S.K. and Srivastava, S.D. 1979. Structure of zizogenin, a new sapogenin from Zizyphus mauritania. Phytochemistry 18, 1758-1759. 